\title{
Duplication cyst of the ileum presenting with severe anemia detected by double-balloon endoscopy
}

\section{다 (i)}

\author{
Authors \\ Suto' ${ }^{1}$, Takanori Goi ${ }^{2}$, Yoshiaki Imamura ${ }^{3}$, Yasunari Nakamoto ${ }^{1}$ \\ Institutions \\ 1 Second Department of Internal Medicine, Faculty of \\ Medical Sciences, Fukui University, Fukui, Japan \\ 2 First Department of Surgery, Faculty of Medical \\ Sciences, Fukui University, Fukui Japan \\ 3 Division of Surgical Pathology, University of Fukui \\ Hospital, Fukui, Japan
}

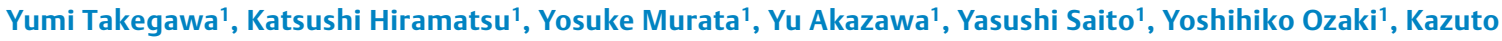
Takahashi' ${ }^{1}$, Tatsushi Naito' ${ }^{1}$ Kazuya Ofuji ${ }^{1}$, Hidetaka Matsuda ${ }^{1}$, Masahiro Ohtani ${ }^{1}$, Tomoyuki Nemoto ${ }^{1}$, Hiroyuki

submitted 28.7.2017

accepted after revision 22.11 .2017

\author{
Bibliography \\ DOI https://doi.org/10.1055/s-0043-125142 | \\ Endoscopy International Open 2018; 06: E395-E398 \\ (c) Georg Thieme Verlag KG Stuttgart · New York \\ ISSN 2364-3722
}

\author{
Corresponding author \\ Yasunari Nakamoto, MD, PhD, Second Department of \\ Internal Medicine, Faculty of Medical Sciences, Fukui \\ University, 23-3 Matsuoka Shimoaizuki, Eiheiji-cho, \\ Yoshida-gun, Fukui 910-1193, Japan \\ Fax: +81-776-61-8110 \\ nakamoto-med2@med.u-fukui.ac.jp
}

\begin{abstract}
Background and study aims Duplication cysts of the ileum are rare and present with non-specific clinical manifestations such as abdominal pain, vomiting, melena, and intussusception. Therefore, preoperative diagnosis is difficult. Here, we report a case of duplication cyst of the small intestine that was diagnosed preoperatively using doubleballoon enteroscopy. A 19-year-old man presented with severe iron deficiency anemia, abdominal pain, and exertional dyspnea. Gastroscopy and colonoscopy revealed no remarkable findings. Abdominal computed tomography revealed a cystic structure in the ileum. Therefore, we performed double-balloon enteroscopy via the anal route. The intestinal tract was bifurcated, with one segment ending in a blind sac containing normal villi and an ulceration. Tc-99 $\mathrm{m}$ pertechnetate scintigraphy showed no accumulation in the lesion. Accordingly, we diagnosed a duplication cyst and suspected that this was the cause of severe anemia. Following small bowel resection with cyst excision and anastomosis, the anemia and presenting symptoms resolved. This report highlights the usefulness of double-balloon enteroscopy of the small intestine for preoperative diagnosis of the obscure gastrointestinal bleeding, including duplication cysts.
\end{abstract}

\section{Introduction}

Duplications of the gastrointestinal tract are rare congenital anomalies that are often found early in life (more than $80 \%$ are diagnosed in children under 2 years of age, and rarely in adults) $[1,2]$. The preoperative diagnosis of duplication cyst is difficult because the clinical manifestations are highly variable, especially in adults. Moreover, duplication cysts sometimes cause obscure gastrointestinal bleeding.

Double-balloon enteroscopy has a prominent role in examination of the small intestine, especially in patients with obscure gastrointestinal bleeding. Double-balloon enteroscopy makes it possible to visualize, sample, and endoscopically treat (using clipping, argon plasma hemostasis, polypectomy, etc.) lesions.
In this case, we performed double-balloon enteroscopy in a patient with severe iron deficiency anemia, and were able to diagnose a duplication cyst preoperatively. We present the patient's unique clinical course, enteroscopy images, and surgical pathology.

\section{Case report}

A 19-year-old man with severe iron deficiency anemia was admitted to our hospital. At age 15, he was diagnosed with anemia during a medical checkup, but was not treated. Three months before admission, he developed abdominal pain and exertional dyspnea. In January 2016, he visited our hospital due to sudden onset of painful paraphimosis. The urologist treated this with manual repositioning and circumcision was 

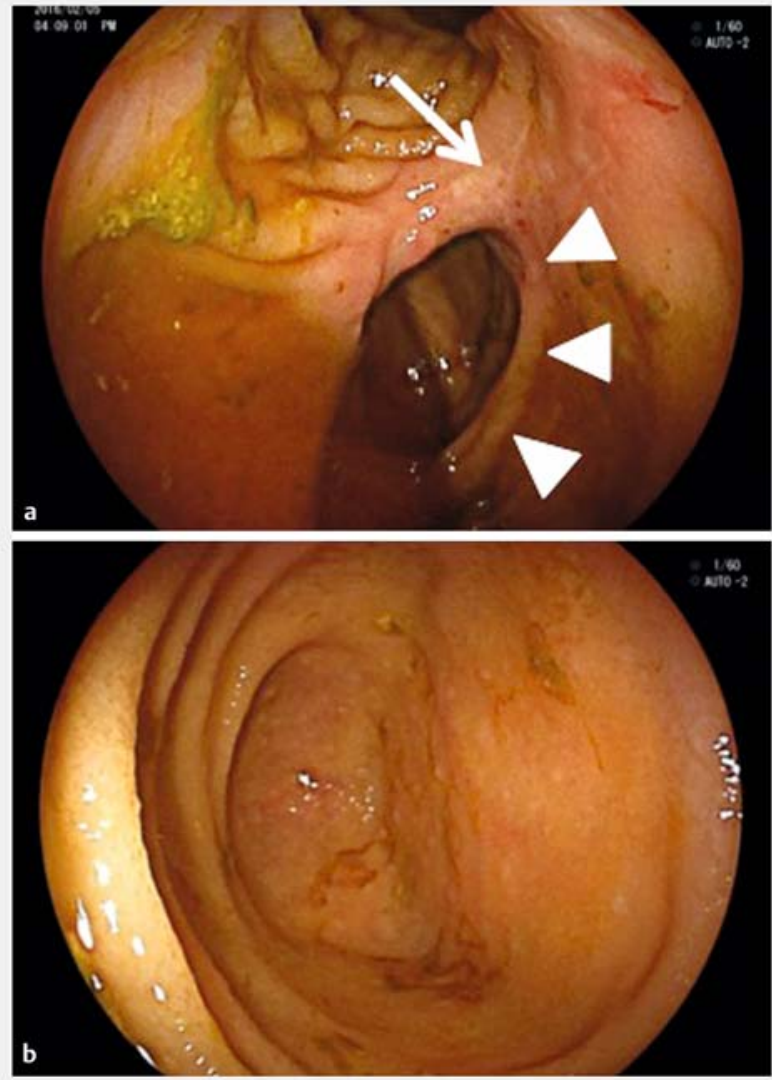

- Fig. 1 Images on double-balloon enteroscopy. a The intestinal tract was bifurcated, with one segment connected to a blind sac (white triangles). A large, shallow, and irregular ulcer near the bifurcation (white arrow) is shown. The ulcer bled easily from contact during endoscopy but there were no exposed vessels at its base. b The mucosal surface of the blind sac was covered with normal villi.

scheduled. Preoperative blood testing revealed severe iron deficiency anemia (red blood cells $2.53 \times 10^{6} / \mathrm{dL}$, hemoglobin $4.3 \mathrm{~g} / \mathrm{dL}$, hematocrit $17.5 \%$, mean corpuscular volume $69.2 \mathrm{fl}$, mean corpuscular hemoglobin $17.0 \mathrm{pg}$, mean corpuscular hemoglobin concentration $24.6 \mathrm{~g} / \mathrm{dL}$ ). The cause of bleeding was not evident on upper gastrointestinal endoscopy or colonoscopy. Abdominal computed tomography was performed and a cystic structure resembling Meckel's diverticulum was found in the ileum. Double-balloon enteroscopy via the anal route was performed and revealed a narrowed distal ileal lumen, $50 \mathrm{~cm}$ from the ileocecal valve. After passage through the narrowed lumen, the intestinal tract was bifurcated ( $>$ Fig.1a), with one

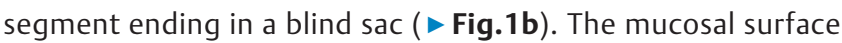
of the blind sac consisted of normal villi. A large, shallow, irregular ulcer was found near the bifurcation ( $\vee$ Fig.1a). The ulcer bled easily on contact during endoscopy but there was no exposed vessel at the base. The blind sac was clearly visible in a small bowel series ( $>$ Fig. 2). Tc-99m pertechnetate scintigraphy was performed for differential diagnosis of Meckel's diverticulum. However, no accumulation was detected in the lesion. Accordingly, we diagnosed a duplication cyst and suspected

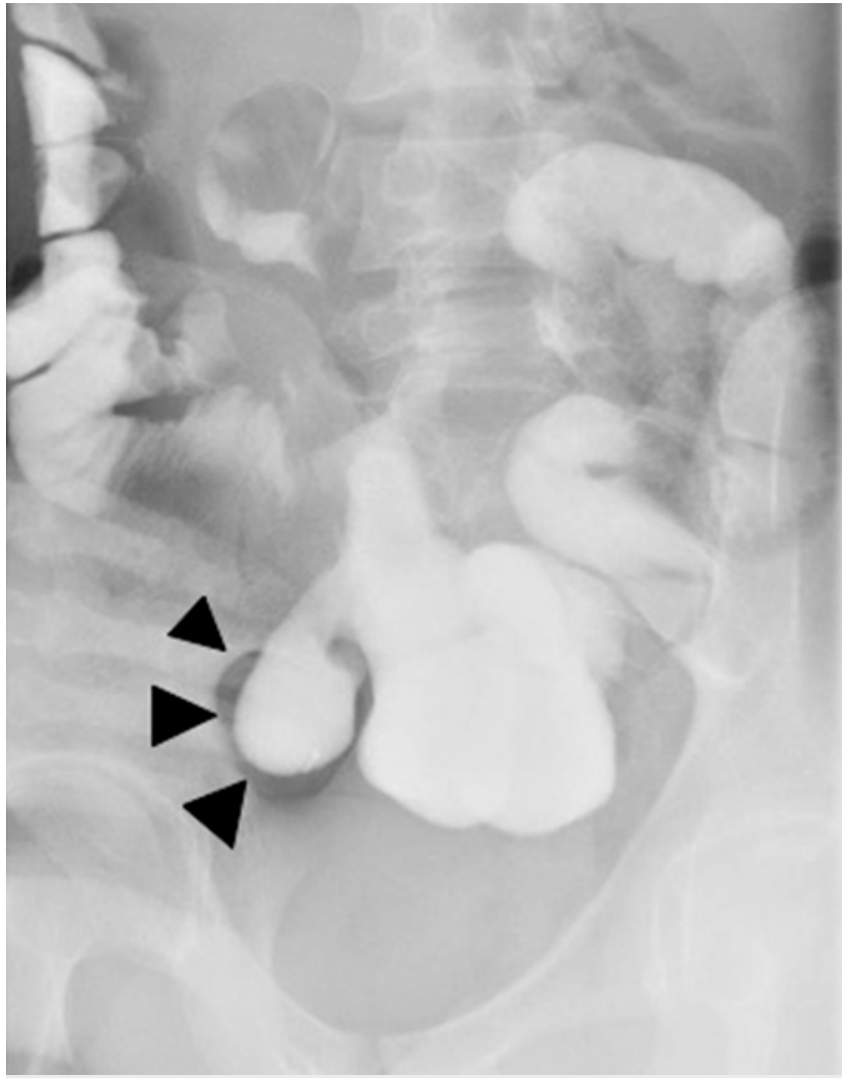

Fig. 2 Images of the small bowel series. The blind sac is clearly visible (black triangles).

that this was the cause of severe anemia. In February 2016, small bowel resection with cyst excision and anastomosis was performed. The anemia and presenting symptoms subsequently resolved. At laparotomy, a bulb-shaped, 4.5-cm cyst was noted on the mesentery that protruded from the ileum ( $>$ Fig.3a). Resection of $30 \mathrm{~cm}$ of ileum included the cyst. The distal side of the cyst was stenotic and the proximal side was dilated ( $\triangleright$ Fig. 3b). The mucosal surface of the cyst had normal villi ( $\triangleright$ Fig.3c). Pathology showed normal intestinal mucosa and muscularis propria, similar to the normal ileal surface ( $\triangleright$ Fig.4a), which was additionally confirmed on a high-magnification image ( $\mathbf{F i g . 4 b}$ ). No ectopic gastric mucosa or pancreatic tissue was observed in the cyst. An ulcer (UL-II: loss of tissue to the submucosal layer) was present near the cyst. The postoperative diagnosis was a duplication cyst of the ileum. Postoperative recovery was uneventful and the patient was discharged 11 days after surgery. He remains symptom-free.

\section{Discussion}

Duplication cysts are rare congenital malformations (1/10,000 live birth) that can appear anywhere in the gastrointestinal tract from the oral cavity to the anus [2-4]. Eighty percent are diagnosed in children below the age of 2 years, with a male predominance $[1,2]$. The ileum is the most frequently affected site [2]. 

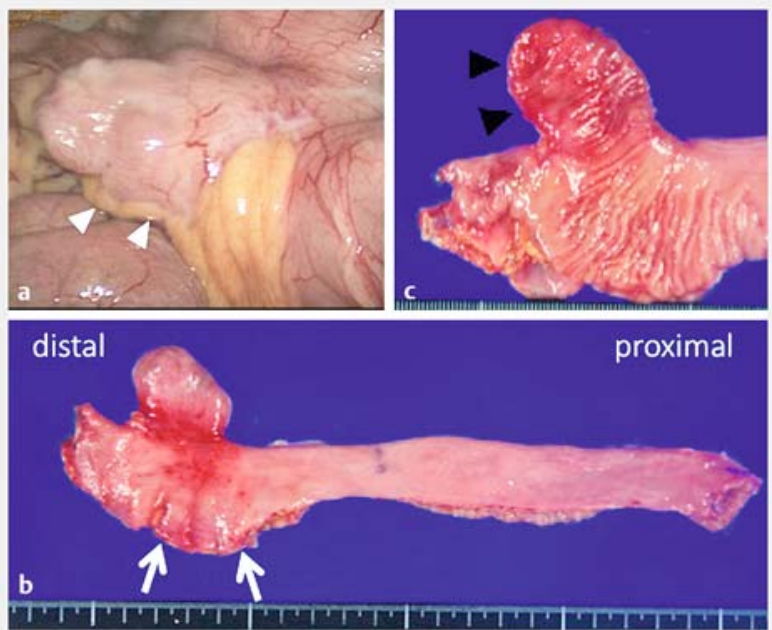

- Fig. 3 Laparoscopic findings during surgical resection and gross findings of the resected specimen. a At laparotomy, a 4.5-cm bulb-shaped cyst was present on the mesentery and protruded from the ileum (white triangles). b Distal ileal resection $(30 \mathrm{~cm})$ included the cyst. Stenosis on the distal side and dilation on the proximal side of the cyst (white arrows). c The mucosal surface of the cyst (black triangles) showing normal villi.

Symptoms include abdominal pain, vomiting, melena, constipation, intussusception, and ileus [1-8]. They can be easily misdiagnosed as other disorders, including appendicitis, Crohn's disease, and Meckel's diverticulum. For this reason, the preoperative diagnosis of a duplication cyst is difficult. Hos- hi et al. reported that correct preoperative diagnostic yield for a duplication cyst was $11.2 \%$ in Japan [7].

Duplication cyst of the ileum must be differentiated from Meckel's diverticulum. Meckel's diverticulum is a true congenital diverticulum derived from the remnant omphalomesenteric duct during development of the terminal ileum, while a duplication cyst can occur anywhere in the gastrointestinal tract, most commonly in the ileum. Meckel's diverticulum is normally located on the antimesenteric aspect, while ileal duplication cysts normally appear on the mesenteric aspect. When a duplication cyst is detected, resection and anastomosis, including that of adjacent normal intestine, is required in all cases, because of the shared common bowel wall and blood supply [4, 9]. All duplications should be surgically treated at the time of diagnosis owing to possible complications including bowel perforation, bleeding, obstruction, and malignant changes. Notably, $23 \%$ of intestinal duplication cysts in adults were affected by ileal cancer $[3,4,9,10]$. In contrast, Meckel's diverticulum has a vitelline artery or a clearly independent blood supply, and most cases require cystectomy alone.

Duplication cysts may cause severe anemia in some cases. Melena was the presenting symptom in $10.5 \%$ of patients with a duplication cyst, and ectopic mucosal tissue was detected in $54.5 \%[6,7]$. Tc-99m pertechnetate scintigraphy is helpful for diagnosis when duplication cysts have ectopic gastric mucosa. In our case, an ulcerated area was present in the duplication cyst, but no ectopic mucosa was detected. Therefore, the ulcer may have been caused by repeated transient intussusception and inflammation around the bifurcation of the sac. This might have explained to long-standing severe anemia and narrowing of the small intestine.

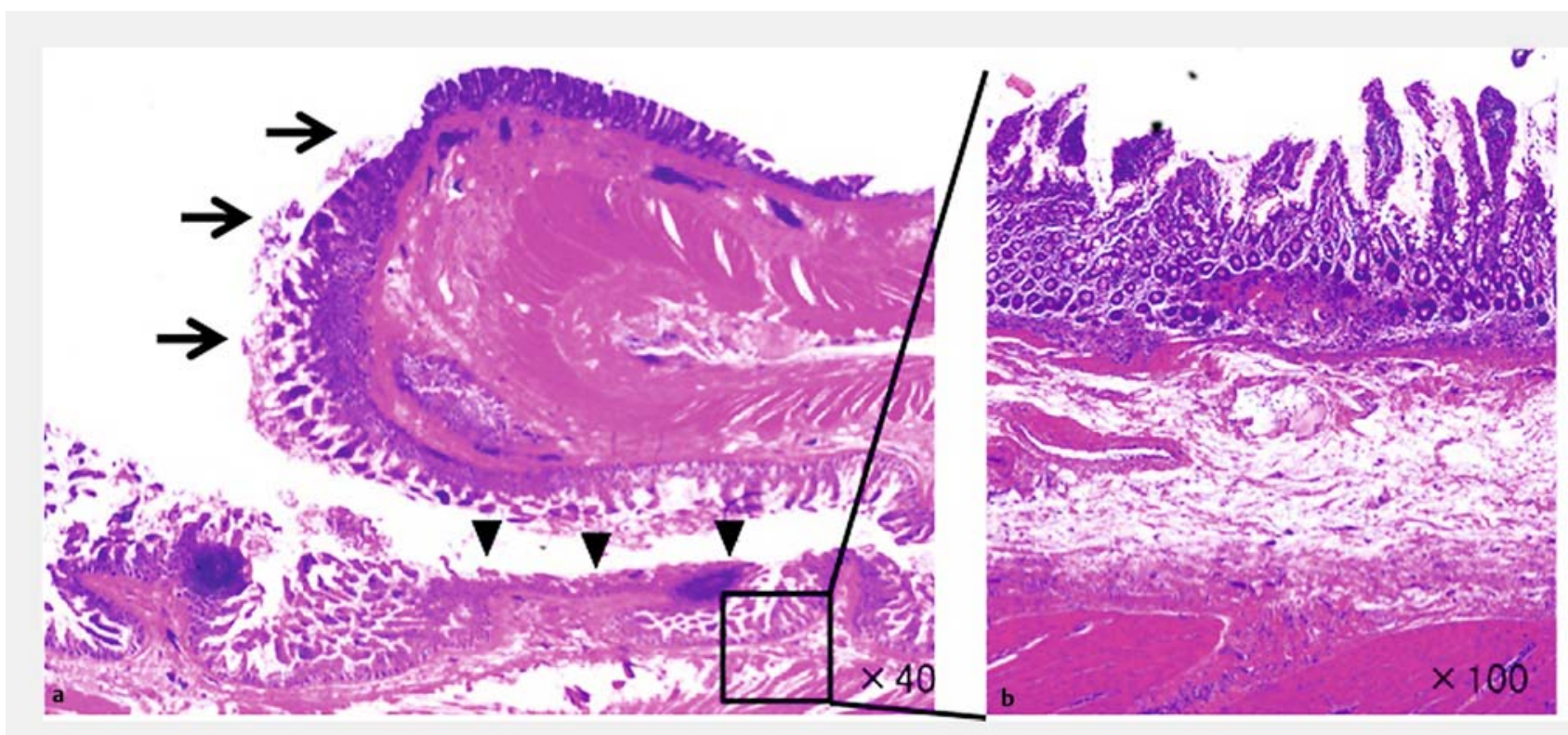

- Fig. 4 Pathological images of the surgical specimen. a Surface of the cyst (black triangles) consisting of normal intestinal mucosa and muscularis propria, as in normal ileum (black arrows, $\times 40$ ). No ectopic gastric mucosa or pancreatic tissue was found. b Normal intestinal mucosa was confirmed on a high-magnification image $(\times 100)$ 


\section{Conclusion}

Duplication cysts are rare in adults, and there are few reports of diagnosis before surgery. We report a case of duplication cyst of the ileum, presenting with severe anemia, diagnosed with double-balloon enteroscopy, and treated without incident. Therefore, the differential diagnosis in a patient with unexplained anemia and abdominal pain should include a possible duplication cyst. Additionally, endoscopic evaluation should be performed.

Competing interests

None

\section{References}

[1] Ekbote G, Pokharkar AB, Moon P. A rare case of perforated tubular ileal duplication in 72-year-old male. Indian J Surg 2013; 75: 418 - 420

[2] Fiorani C, Scaramuzzo R, Lazzaro A et al. Intestinal duplication in adulthood: A rare entity, difficult to diagnose. World J Gastrointest Surg 2011; 3 : $128-130$
[3] Gjeorgjievski M, Manickam P, Ghaith G et al. Safety and efficacy of endoscopic therapy for nonmalignant duodenal duplication cysts: Case report and comprehensive review of 28 cases reported in the literature. Medicine (Baltimore) 2016; 95: e3799

[4] Li BL, Huang X, Zheng C] et al. Ileal duplication mimicking intestinal intussusception: a congenital condition rarely reported in adult. World J Gastroenterol 2013; 19: 6500-6504

[5] Yamasaki A, Onishi H, Yamamoto H et al. Asymptomatic adenocarcinoma arising from a gastric duplication cyst: A case report. Int J Surg Case Rep 2016; 25: 16 - 20

[6] Tsuji H, Kumagai M, Inada Y et al. Usefulness of video capsule endoscopy and double-balloon endoscopy in the preoperative diagnosis of a jejunal duplication cyst. Gastroenterol Endosc 2014; 56: 457-464

[7] Ogino H, Ochiai T, Nakamura $\mathrm{N}$ et al. Duplication cyst of the small intestine found by double-balloon endoscopy: a case report. World J Gastroenterol 2008; 14: $3924-3926$

[8] Yu Y, Wu JS, Ke ZW. Giant chylous cyst and ileal duplication in a young adult. Singapore Med J 2014; 55: e77-81

[9] Hamza AR, Bicaj BX, Kurshumliu Fl et al. Mesenteric Meckel's diverticulum or intestinal duplication cyst: A case report with review of literature. Int J Surg Case Rep 2016; 26: 50 - 52

[10] Kusunoki N, Shimada Y, Fukumoto $S$ et al. Adenocarcinoma arising in a tubular duplication of the jejunum. J Gastroenterol 2003; 38: 781 785 